\section{Introduction au colloque}

Catherine Paradeise

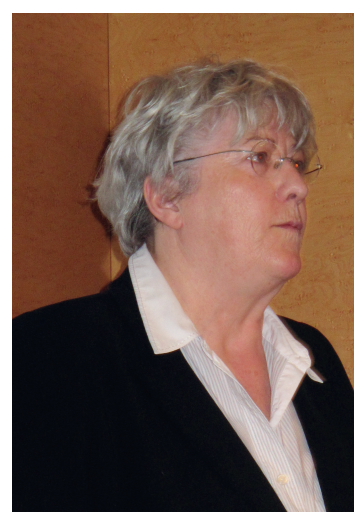

IFRIS (Institut francilien

Recherche, Innovation, Société), Université Paris Est-LATTS,

LATTS-département de sociologie, bâtiment Lavoisier,

cité Descartes,

5 , boulevard Descartes,

Champs-sur-Marne

77454 Marne-la-Vallée Cedex 2, France.

catherine.paradeise@univ.mlv.fr
Pierre Tambourin et moi-même, nous nous connaissons depuis longtemps, je dirai même qu'il y a de la complicité entre nous, ce qui a favorisé la mise en place de cette série de cinq colloques. Ceux-ci ont débuté en 2010. Nous avions l'idée d'une part que la science en société était le bout par lequel il fallait aborder la question des relations sciences/société et d'autre part, nous étions convaincus que, dans cette entreprise et dans les bouleversements à la fois de la science et de la société que nous vivons, le dialogue entre les sciences de l'homme et de la société et les sciences de la vie était une nécessité difficile à satisfaire, car le monde des sciences de la vie peut écouter le monde des sciences de l'homme et de la société, mais avec quelques difficultés... Et réciproquement ! Aussi est-il difficile de construire un dialogue approfondi entre ces deux mondes.

Pour approfondir cette question de la science en société et pour essayer d'aller plus loin, d'amorcer ce dialogue en profondeur entre les sciences de l'homme et de la société et les sciences de la vie, nous avons souhaité mettre en place ces cinq colloques consécutifs; celui-ci est le deuxième.

L'an dernier nous avons abordé la question en quelque sorte de l'extérieur, en regardant les différents types de publics de la science, voire les publics introuvables des sciences émergentes, avec l'idée qu'il fallait tenter de «se départir d'une position qui opposerait des scientifiques rationnels à une société en proie à ses émotions et à ses peurs pour comprendre comment science et société sont co-construites ».

Cette année, nous proposons d'aller plus loin en nous intéressant à la façon dont cette science est construite et fabriquée. Quelles manières de connaître ? Quels instruments cognitifs et matériels ? Ces divers types d'instruments - épistémiques pour les uns, techniques pour les autres - ne sont pas complètement étrangers les uns aux autres. Les dynamiques d'évolution croisée entre les épistémologies scientifiques et les instrumentations scientifiques méritent d'être étudiées de près, en particulier en considérant combien la biologie a connu de bouleversements au cours des 20 ou 30 dernières années.

Le plaisir intellectuel ne suffit pas à soutenir la production de connaissance. Encore faut-il penser et faire valoir que la connaissance est produite en société et entre en société. Traditionnellement, la science est conçue comme ouverte. Pourtant, depuis longtemps, mais d'une manière accélérée au tournant $d u x x^{e}$ siècle, la science est aussi entrée « en marché ». Il importe donc de comprendre comment la marchandisation de la science a un impact à la fois sur les manières de connaître et l'instrumentation de la science, et comment les manières de connaître, l'instrumentation de la science, la mise en marché peuvent poser problème ou peuvent aider à résoudre un certain nombre de problèmes de cette science et de sa vie en société.

Le deuxième pari que nous faisions avec Pierre Tambourin - et que nous espérons mieux affronter cette année que l'année dernière - était de rapprocher vraiment, dans un dialogue construit en profondeur à partir de l'observation d'objets scientifiques, chercheurs des sciences de la vie (SDV) et chercheurs des sciences de l'homme et de la société (SHS). Le colloque de l'année dernière a été apprécié. Cependant, nous avons constaté que les chercheurs des sciences de l'homme et de la société y ont beaucoup accaparé le temps de parole. On sait que les chercheurs SHS sont des «beaux parleurs », quelquefois on pense même dans le monde des SDV accaparé par ses humbles travaux à la paillasse, qu'ils parlent trop bien pour être parfaitement honnêtes! Cette année, nous avons souhaité faire évoluer cette vision, d'une part en construisant mieux la relation à l'amont de cette journée, à travers des ateliers de préparation de chacune des trois sessions, et d'autre 
part en réalisant un meilleur équilibre à la tribune entre chercheurs des sciences de l'homme et de la société et chercheurs des sciences de la vie. Nous verrons aujourd'hui si ce pari est couronné de succès. J'espère que cela fonctionnera et que dans les trois prochaines années, nous irons encore plus loin dans ce dialogue au bénéfice mutuel, en continuant la série avec une journée autour des problèmes liés au développement de la biologie de synthèse, sujet complexe et polémique. Dès la fin de ce colloque, nous nous remettrons donc au travail pour produire les actes et pour préparer cette prochaine journée.

J'ai commencé en évoquant une préoccupation commune à Pierre Tambourin et moi-même. À dire vrai, il s'agit plutôt d'une préoccupation commune à deux institutions, Genopole ${ }^{\circledR}$ et IFRIS. L'IFRIS, récemment promu «laboratoire d'excellence » et «DIM ${ }^{1} »$, est en pleine effervescence. Nos

${ }^{1}$ DIM : domaine d'intérêt majeur. deux institutions ont eu le désir de porter ce mouvement. Je souhaite qu'elles le poursuivent, démontrant ainsi en marchant combien elle est fructueuse, et peut-être même indispensable, pour nos deux secteurs disciplinaires. $\diamond$
TIRÉS À PART

C. Paradeise 